\title{
Health economic impact of a biopsy-based cell cycle gene expression assay in localized prostate cancer
}

\author{
Gary Gustavsen*,1 (iD), Kelsey Taylor ${ }^{1}$, Doria Cole ${ }^{1}$, Laura Gullet ${ }^{1}$ \& Nicolas Lewine ${ }^{1}$ \\ ${ }^{1}$ Health Advances LLC, Newton, MA 02466, USA \\ *Author for correspondence: Tel.: +1 781647 3435; Fax: +1 781392 1484; ggustavsen@healthadvances.com
}

\begin{abstract}
Background: Prior studies have established that broader incorporation of active surveillance, guided by additional prognostic tools, may mitigate the growing economic burden of localized prostate cancer in the USA. This study sought to further explore the potential of a particular gene expression-based prognostic tool to address this unmet need. Materials \& methods: A deterministic, decision-analytic model was developed to estimate the economic impact of the Prolaris ${ }^{\circledR}$ test on a US commercial health plan. Results \& conclusion: When adopted in patients classified by the American Urological Association as low or intermediate risk, the assay was projected to reduce costs by $\$ 1894$ and $\$ 2129$ per patient over 3 and 10 years, respectively, largely through the increased use of active surveillance.
\end{abstract}

First draft submitted: 24 June 2020; Accepted for publication: 12 August 2020; Published online: 9 September 2020

Keywords: biomarkers/oncogenes/molecular oncology $\bullet$ health economics $\bullet$ pharmacoeconomics $\bullet$ prognosis • radiation therapy/radiotherapy $\bullet$ real-world evidence $\bullet$ screening $\bullet$ surgery $\bullet$ surveillance $\bullet$ urologic/prostate

As the most common malignancy affecting American men, prostate cancer represents a substantial portion of overall cancer spending in the USA today and a significant financial burden on the healthcare system [1]. A study by researchers at the National Cancer Institute projected the costs of prostate cancer care to grow from $\$ 11.85$ billion in 2010 to $\$ 16.34$ billion by 2020 [2]. Increasing in cost more quickly than any other cancer, this ranks prostate cancer as the third most costly cancer overall, behind breast and colorectal cancer [2].

A significant driver of the treatment costs associated with localized prostate cancer is the widespread use of immediate, definitive treatment for the majority of patients upon diagnosis. Regardless of disease aggressiveness, most patients today (75-90\%) receive immediate, definitive treatment for their disease, which typically includes radical prostatectomy (RP), radiation therapy (RT) androgen deprivation therapy (ADT), or some combination of these [3-7]. However, it is widely recognized that a substantial subset of patients could live with their indolent disease and ultimately die from other causes, making definitive treatment an unnecessary route for many patients [8]. In addition, all current treatment options entail side effects that affect quality of life [9]. Meanwhile, the cost of these treatments is substantial, with RT alone costing payers more than $\$ 34,000$ per patient $[10,11]$. Recent studies have demonstrated that active surveillance (AS) is a suitable, less aggressive treatment option for many patients with less aggressive localized prostate cancer and one that can reduce the overall cost of patient care. However, determining which patients are best suited for AS remains a challenge, especially for intermediate-risk patients [12].

Patients who have been newly diagnosed with prostate cancer may have either indolent or aggressive forms of the disease. Unfortunately, current tools such as prostate-specific antigen levels, Gleason scores and nomograms do not adequately stratify patients within American Urological Association (AUA) risk groups [13]. Today, many men receive unnecessary treatment for their disease, as evidenced by low rates of prostate cancer-specific mortality, even among untreated patients [8]. Improved stratification based on disease aggressiveness and mortality risk has the potential to spare men with indolent disease from unnecessary treatment. This enhanced triaging has the potential to improve clinical outcomes through more appropriate treatment of patients, reduce economic inefficiencies and potentially reduce costs. Several molecular assays have emerged to help physicians triage patients. However, the 
overall cost-effectiveness of these tools remains unknown given the high costs of these tests and variability in the upfront and long-term costs associated with managing patients who receive more or less aggressive treatment.

The Prolaris ${ }^{\circledR}$ test (Myriad Genetics, UT, USA) is a gene expression-based assay that measures tumor cell growth characteristics in order to stratify patients with localized prostate cancer according to disease aggressiveness. The test combines the gene expression levels of 31 genes related to cell cycle progression and 15 housekeeping genes into a Cell Cycle Progression score that is used to predict 10-year prostate cancer-specific disease mortality and 10-year probability of progression to metastatic disease. The assay's prognostic ability at biopsy has been evaluated in 14 published clinical trial cohorts of prostate biopsy and prostatectomy samples [14-25]. These studies have validated the ability of the test to identify both lower-risk patients who would be better suited to more conservative options such as AS and higher-risk patents who would benefit from definitive treatment beyond clinicopathologicbased recommendations. A combined clinical Cell Cycle Risk (CCR) score has been developed that incorporates both prognostic molecular and clinicopathologic information. Building on these data, a threshold score has been validated [15] that optimally identifies men with low-risk disease for whom AS would be appropriate based on a 10 -year risk of disease-specific mortality of $3.2 \%$ [22].

Separate studies have evaluated the assay's clinical utility in terms of the ability of the test to impact clinical decision-making [26,27]. Results from prospective clinical utility registries demonstrated that the test had a significant impact on the management of patients with prostate cancer, particularly by increasing the use of AS for both lowand intermediate-risk patients. These studies evaluated 1537 total patients from geographically diverse sites. Furthermore, real-world examples have emerged supporting the utility of stratification tools such as Prolaris to increase the use of AS; when Blue Shield of California began to cover Prolaris testing, it found that AS increased by $18 \%$, radical prostatectomies decreased by $8 \%$ and intensity-modulated radiation therapy decreased by $14 \%$ [28].

Given the recent results supporting the utility of Prolaris for guiding appropriate stratification of patients for AS, it is important to assess the downstream economic impact of this test on payers - particularly given the current economic environment and significant costs associated with prostate cancer care [29]. An increase in the use of AS in the management of low- and intermediate-risk patients associated with using the cell cycle gene expression assay could have a profound impact on the cost of prostate cancer care. The purpose of this study was to model the economic impact of the test on a hypothetical US commercial insurer using an in silico model.

\section{Materials \& methods}

Model structure \& methodology

A deterministic, decision-analytic model was developed to estimate the direct medical costs associated with using the cell cycle gene expression assay compared with standard clinicopathologic evaluation alone to guide the management of patients with localized prostate cancer. A probabilistic-model approach with 1 year intervals was deemed optimal in this case given the level of complexity and data availability. Treatment of a single hypothetical cohort of incident-localized prostate cancer patients was simulated using a patient flow model, built in Microsoft Excel, that was structured according to prevailing management protocols, including AUA and National Comprehensive Cancer Network (NCCN) guidelines. Two branches of the model represented patient management under different scenarios. The first branch used standard clinicopathologic diagnostic modalities alone to determine patient management (the 'typical practice scenario') and the second branch incorporated the cell cycle gene expression assay (the 'Prolaris Scenario'). As is common in clinical practice, the hypothetical patient cohort was separated into cohorts based on AUA risk groups. These groups are substantially similar to the NCCN risk group classifications, which are also often used by clinicians to stratify patents. Patients assigned to low- and intermediate-risk groups were modeled to receive the test after a biopsy, in order to determine whether to pursue AS or definitive treatment. The hypothetical patient cohort focused on AUA low- and intermediate-risk patients given the demonstrated clinical impact of Prolaris to increase the use of AS in these specific cohorts. Patients in the AUA high-risk group were not modeled in this study because AS is not prevalent in the management of these patients.

Patient flow was modeled over the course of 10 years, tracking each phase of care across the cohort. Costs were assigned to each unit of care based on estimated average reimbursement rates paid by a US commercial health plan, as described below. A comparison of the overall cost of managing patients under the typical practice scenario versus the Prolaris Scenario was used to determine the economic impact of the gene expression assay. A graphical schematic of the model structure is depicted in Supplementary Figure 1. 
Table 1. Clinical treatment paradigm for the typical practice scenario.

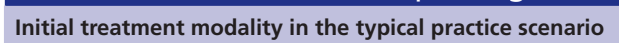

Initial treatment modality in the typical practice scenario
AUA risk group

Intermediate $(35 \%$ of patients with localized prostate cancer)

Active surveillance $(\%)$

Radical prostatectomy only (\%)

Radiation therapy only (\%)

Androgen deprivation therapy only (\%)

Radiation therapy and androgen deprivation therapy (\%)

Total (\%)

Low $(42 \%$ of patients with localized prostate cancer)

24

36

25

15

100
40

10

29

17

100

Initial treatment modality of patients with prostate cancer by AUA risk group. The typical practice scenario represents the modeled average treatment paradigm using standard clinicopathologic diagnostic modalities alone to determine patient management. The clinical practice paradigm was based on a combination of relevant sources, including published clinical guidelines, peer-reviewed articles on current treatment of patients with prostate cancer and in-depth qualitative interviews with board-certified physicians. Primarily, data from the US

National Cancer Database drove the active surveillance assumptions and data from the AUA Quality Registry drove the subsequent treatment breakdown.

ADT: Androgen deprivation therapy; AS: Active surveillance; AUA: American Urological Association; RP: Radical prostatectomy; RT: Radiation therapy.

\section{Clinical paradigm}

Typical practice scenario

The typical practice scenario builds upon a recently published study that utilized a decision-analytic model to estimate the medical costs associated with localized prostate cancer over 10 years for all AUA risk groups, from the perspective of a US commercial payer [30]. The key assumptions regarding the initial staging and treatment of incident-localized, AUA low- and intermediate-risk prostate cancer patients in the typical practice scenario are presented in Table 1. Table 1 maps out the initial care received by each patient cohort based on AUA risk group. Additional follow-up care, as well as progression and further treatment costs, were tracked over the 10-year duration of the model to follow patients through the care continuum (see 'Disease follow-up and progression' section). Patients initially managed with AS were modeled to subsequently receive definitive treatment according to published rates (roughly $30 \%$ of low-risk and $60 \%$ of intermediate-risk patients over 5 years), either due to disease progression or patient choice [31-33].

The current clinical practice paradigm was based on a combination of relevant sources, including published clinical guidelines, peer-reviewed articles on current treatment for patients with prostate cancer and in-depth qualitative interviews with board-certified physicians. Data from the US National Cancer Database were used to inform baseline rates of AS, whereas data from the AUA Quality Registry served as the foundation for the treatment distribution across definitive treatment modalities [34,35]. A total of 23 board-certified urologists were interviewed about their current practice patterns and treatment paradigms to further inform the patient management paradigm. All urologists who were interviewed had been in practice for at least 5 years and were actively treating patients with prostate cancer. The urologists practiced in a mix of community and academic settings from all geographic regions in the USA. No two physicians practiced at the same institution. An in-depth review of the clinical literature, including studies on the National Cancer Database and AUA Quality Registry, was used to validate and refine physician perspectives on the management of patients with localized prostate cancer.

The options modeled for initial patient management were AS, single-modality definitive treatment (including $\mathrm{RP}, \mathrm{RT}$ or primary ADT) or multimodality definitive treatment (RT plus ADT).

The clinical paradigm (Table 1) was intended to represent today's prevailing practice patterns. Accordingly, initial patient management varied by AUA risk group. In the AUA low-risk group, $24 \%$ of patients were initially managed using AS, whereas $76 \%$ received single-modality therapy. In the AUA intermediate-risk group, $4 \%$ were initially managed using AS, 79\% with single-modality therapy and $17 \%$ with multimodality therapy $[31,32,36-40]$.

\section{Prolaris scenario}

The impact of the Prolaris test on patient management and the use of AS was determined based on the published results of the AS Threshold Validation Study by Lin et al., as well as taking into account adherence from the Outcomes of Men Who Select AS study, as shown in Table 2 [22,41]. Lin et al. validated the threshold for AS eligibility and determined that, on average, $92.9 \%$ of AUA low-risk patients and $43.0 \%$ of AUA intermediate-risk patients are eligible for AS based on having a CCR score and therefore a 10-year prostate cancer mortality risk, below the threshold value [22]. To account for variability in real-world practice, an adherence rate of $82.4 \%$ was 
Table 2. Active surveillance rate by risk group in the prolaris scenario.

\begin{tabular}{|c|c|c|}
\hline \multirow[t]{2}{*}{ Parameters } & \multicolumn{2}{|c|}{ AUA risk group } \\
\hline & Low & Intermediate \\
\hline Eligible for active surveillance (\%) & 92.9 & 43.0 \\
\hline Adherence rate to a recommendation of active surveillance (\%) & 82.4 & 82.4 \\
\hline Rate of active surveillance in the Prolaris Scenario (\%) & 76.5 & 35.4 \\
\hline \multicolumn{3}{|c|}{$\begin{array}{l}\text { Calculation for the rate of active surveillance in Prolaris Scenario by risk group. The active surveillance rate in the Prolaris Scenario was determined based on published results demonstrating } \\
\text { the fraction of AUA risk groups eligible for active surveillance. Results were based on the validated cell cycle risk score and adjusted based on published rates of adherence to a } \\
\text { recommendation of active surveillance }[22,41] \text {. } \\
\text { AUA: American Urological Association. }\end{array}$} \\
\hline
\end{tabular}

Table 3. Clinical treatment paradigm in the prolaris scenario.

\begin{tabular}{|c|c|c|}
\hline \multirow[t]{2}{*}{ Parameters } & \multicolumn{2}{|c|}{ AUA risk group } \\
\hline & Low & Intermediate \\
\hline Active surveillance (\%) & $76.5(82.4 \%$ adherence to AS recommendation rate of $92.9 \%)$ & $35.4(82.4 \%$ adherence to AS recommendation rate of $43 \%)$ \\
\hline RP only (\%) & 11.1 & 24.4 \\
\hline RT only (\%) & 7.7 & 6.0 \\
\hline ADT only (\%) & 4.7 & 17.7 \\
\hline RT and ADT (\%) & 0 & 16.5 \\
\hline Total $(\%)$ & 100 & 100 \\
\hline
\end{tabular}

applied to the threshold, based on the Outcomes of Men Who Select AS study by Kaul et al., which found that $82.4 \%$ of men with prostate cancer who were determined to have a low estimated risk of disease-specific mortality based on their CCR score and NCCN guidelines initially selected AS over definitive treatment [41]. This translated to a greater than threefold increase in the use of AS in AUA low-risk patients, increasing AS from 24\% in the typical practice scenario to $76.5 \%$ in the Prolaris Scenario. In intermediate-risk patients, using the same adherence rate translated to a greater than eightfold increase in AS use in intermediate-risk patients, from $4 \%$ in the typical practice scenario to $35.4 \%$ in the Prolaris Scenario (Table 2). Similar to the typical practice scenario, patients initially managed with AS were modeled to subsequently receive definitive treatment according to published rates (roughly $30 \%$ of low-risk and $60 \%$ of intermediate-risk patients over 5 years), either due to disease progression or patient choice [31-33]. Initial treatment for those men not receiving AS was distributed proportionally based on the treatment paradigm used in the typical practice scenario, as highlighted in Table 3.

\section{Disease follow-up \& progression}

Patients were modeled to receive follow-up care and additional treatment based on standard clinical practice. Patient follow-up care and disease monitoring after initial management includes office visits, prostate-specific antigen screening and biopsies, as well as treatment for complications resulting from management, such as erectile dysfunction and incontinence. A declining annual rate of biochemical recurrence was modeled according to rates in the published literature and varied based on initial treatment type and AUA risk group [10,37]. Patients who experienced biochemical recurrence were modeled to receive additional therapy, with patients initially treated using single-modality RP going on to receive either salvage RT $(50 \%)$ or ADT $(50 \%)$ and patients initially treated with all other modalities going on to receive ADT [42]. For patients progressing to later stages of the disease, the rate of progression was based on published statistics. The timeline for patients experiencing progression was based on published survival statistics and estimated progression curves established during physician interviews [43].

\section{Cost inputs}

Table 4 displays the unit costs used to populate the economic model. The cost inputs and methodology used in this study build upon a prior study that utilized a decision-analytic model to estimate the medical costs associated with localized prostate cancer over 10 years for all AUA risk groups [30]. Cost inputs were established for each unit 


\begin{tabular}{|c|c|c|c|}
\hline & Cost (USD) & Source & Ref. \\
\hline \multicolumn{4}{|l|}{ Initial treatment } \\
\hline - Radical prostatectomy & $\$ 10,604$ (year 1) & 2018 Medicare fee schedules and claims databases & \\
\hline - Primary radiation therapy & $\$ 34,116$ (year 1) & Voigt (2017) J. Urol. & [11] \\
\hline - Androgen deprivation therapy & $\$ 2993$ (year 1) & 2018 Medicare fee schedules and claims databases & \\
\hline - Adjuvant/salvage radiation therapy & $\$ 29,101$ (year 1) & Voigt (2017) J. Urol. & [11] \\
\hline \multicolumn{4}{|l|}{ Monitoring costs } \\
\hline - Active surveillance & $\$ 1066$ (annual) & 2018 Medicare fee schedules and claims databases & \\
\hline - Post-RP/RT monitoring & $\$ 774-845$ (annual) & $\begin{array}{l}2018 \text { Medicare fee schedules and claims databases } \\
\text { Cooperberg (2013) BJU Int. } \\
\text { Resnick (2013) NEJM }\end{array}$ & {$[10,44]$} \\
\hline Advanced treatment & & 2018 Medicare fee schedules and claims databases & \\
\hline - Androgen deprivation therapy & $\$ 2993$ (annual) & 2018 Medicare fee schedules and claims databases & \\
\hline - Castrate-resistant prostate cancer & $\$ 122,323$ (annual) & 2018 Medicare fee schedules and claims databases & \\
\hline $125 \%$ & \multicolumn{2}{|l|}{ MEDPAC } & [45] \\
\hline \multicolumn{4}{|c|}{$\begin{array}{l}\text { Cost inputs used in the model. Costs were referenced from a variety of sources, including published, peer-reviewed clinical articles and Medicare fee schedules and claims } \\
\text { databases. } \\
\text { ADT: Androgen deprivation therapy; AS: Active surveillance; CRPC: Castrate-resistant prostate cancer; RP: Radical prostatectomy; RT: Radiation therapy. }\end{array}$} \\
\hline
\end{tabular}

of care a patient might undergo, including diagnostic procedures, surgical procedures, radiotherapy procedures and pharmacological therapy. Other costs associated with the treatment, such as office visits, anesthesiology, pathology and associated complications, were accounted for where appropriate. Cost assumptions were generated from a variety of sources given the known variability in payment rates across US commercial health plans. Sources for costs included the 2018 Medicare fee-for-service rates, peer-reviewed journal articles and other published sources. As many of these cost inputs will change over time, results of this study are most relevant from a 2018 perspective.

To estimate the pricing based on Medicare fee-for-service rates, interviews were conducted with professional coders specializing in urology coding to determine which Current Procedural Terminology (CPT), International Statistical Classification of Diseases and Health Problems (ICD-9) and Diagnosis-Related Group (DRG) codes were most commonly used in practice. The CPT, ICD-9 and DRG codes were mapped to national payment rates using 2018 Medicare fee schedules. For each code, the total Medicare reimbursement (combining both professional and facility fees, where appropriate) was calculated for four separate 'place of service' settings as follows: physician's office, ambulatory surgical center, hospital outpatient and hospital inpatient. For procedures performed in a variety of settings, the payment amount(s) for each setting were then combined in a weighted average according to the frequency with which the relevant code was billed from each of the four settings. Data for the CPT codes were sourced from the 2015 Physician/Supplier Procedure Summary (PSPS) database, which contains data on fee-forservice claims billed to Medicare part B. In cases where multiple CPT, ICD-9 or DRG codes were used to describe similar services, a single payment amount was calculated by performing a weighted average of the various codes according to their billing frequency, as recorded in the PSPS or the Nationwide Inpatient Sample, Healthcare Cost and Utilization Project, Agency for Healthcare Research and Quality. Data for the ICD-9 and DRG codes were sourced from the 2014 Nationwide Inpatient Sample. Despite the multiyear data-release lag time, this database contains the largest collection of data on procedures and diagnosis counts from publicly available payer healthcare databases, including Medicare. For the CPT codes, the same PSPS database was used. Finally, Medicare payment rates were inflated by $25 \%$ to more accurately reflect the rates paid by commercial insurers, with the exception of payment rates for pharmaceuticals [46].

Select cost inputs were determined from alternate sources. The cost inputs for certain specific therapies, including primary and adjuvant radiation therapy, were taken from the published literature [11]. The costs of oral pharmaceuticals were sourced from published wholesaler acquisition cost prices and Medicare average sales-price drug pricing. The cost of the gene expression test was sourced from the manufacturer.

The cost of treating castrate-resistant prostate cancer was estimated using a hypothetical treatment regimen based on standard clinical practice (Supplementary Table 1). The analysis accounted for the percentage of patients receiving a given therapy, the duration of therapy and dosing schedule and the average cost of therapy. The cost per milligram was translated into the cost of a full course of treatment according to the dosing schedule specified by 
Table 5. Total cumulative costs per patient over 10 years.

\begin{tabular}{|c|c|c|c|c|}
\hline \multirow[t]{2}{*}{ Parameters } & \multicolumn{4}{|c|}{ Cumulative cost per patient at year after diagnosis } \\
\hline & Year 1 & Year 3 & Year 5 & Year 10 \\
\hline Typical practice scenario & $\$ 17,114$ & $\$ 20,796$ & $\$ 27,872$ & $\$ 70,074$ \\
\hline Prolaris scenario & $\$ 13,123$ & $\$ 18,902$ & $\$ 26,159$ & $\$ 67,945$ \\
\hline Total cumulative cost-savings per patient & $\$ 3991$ & $\$ 1894$ & $\$ 1713$ & $\$ 2129$ \\
\hline
\end{tabular}

Cumulative costs of prostate cancer management in each modeled scenario on a per patient basis in the typical practice scenario and Prolaris Scenario. Prostate cancer management was modeled for all AUA low- and intermediate-risk patients within a typical payer plan. Cumulative costs were compared between scenarios to determine the overall cost-savings per patient. AUA: American Urological Association.

the drug's US FDA label, clinical guidelines, or the most relevant clinical trials. For dosing schedules dependent on body weight or surface area, an average body weight of $88.8 \mathrm{~kg}$ and average body surface area of $1.9 \mathrm{~m}^{2}$ was used, as reported by the CDC in 2016 [45]. A final average per-patient cost of treating castrate-resistant prostate cancer was calculated and validated using various published cost analyses [42,47-52].

Cost-savings analysis

The cumulative costs of the typical practice scenario and the Prolaris Scenario were compared in order to determine the economic impact of the assay on a hypothetical commercial payer. Cost impact was calculated on a 'per patient tested' and a 'per member per month' basis and as the 'total health plan savings', for commercial health plans ranging in size from one to 5 million members. It is important to note that cost-savings results in the model are directly comparable year to year as no discount rate was used in this model.

\section{Sensitivity analyses}

To assess the model's sensitivity to changes in specific inputs, Individual model assumptions on the clinical treatment paradigm and cost inputs were varied across a range of plausible values and the overall cost-savings per patient over 3 years was recalculated. For this univariate analysis, each model input was changed in a way that lowered the cost-savings ('financially conservative') and in a way that increased cost-savings ('financially aggressive'). The assay's cost impact per patient over the first 3 years was reassessed using the minimum and maximum plausible value of each major assumption.

\section{Results}

Overall savings

The average cumulative cost of treating a patient with localized prostate cancer at Year 10 in the typical practice scenario was approximately $\$ 70,074$ per patient. In the Prolaris Scenario, the average cumulative cost was approximately $\$ 67,945$ - a savings of $\$ 2129$ per patient tested after accounting for the cost of the test (Table 5). Throughout the duration of the model, there was no year in which the Prolaris Scenario resulted in increased cumulative costs compared with the typical practice scenario, meaning that using the test results in savings to the payer regardless of the payer's economic time horizon (Table 5).

The economic impact varied across each AUA risk group. The AUA low-risk group generated the greatest costsavings due to a significant reclassification of patients into management with AS. Although the AUA intermediaterisk group also generated significant cost-savings because many patients initially switched to AS, these savings were eroded over time as the majority of those patients ultimately chose definitive treatment. Table 6 illustrates this cost-savings dynamic over time for each risk group, as well as the overall cost-savings per patient after taking into account the relative distribution of AUA risk groups in the general population.

Based on cited epidemiological rates, a typical commercial health plan of 5 million members can expect to insure approximately 3078 patients who receive new prostate cancer diagnoses per year [53]. Use of the test in all AUA low- and intermediate-risk patients would result in approximately 2370 patients tested. Commercial insurers are most focused on the impact of an intervention over the first 3 years, given the frequency with which patients change insurance plans. On average, after 3 years $>50 \%$ of members will have changed or left a typical plan [54,55]. Thus, the first 3 years was selected for assessing the economic impact on a typical commercial insurer. The model demonstrated that testing these patients would result in approximately $\$ 4.5$ million in savings over 3 years. This equates to approximately $\$ 0.11$ in savings per member, per month for the health plan. Table 7 summarizes the 
Table 6. Cumulative cost-savings per patient by risk group.

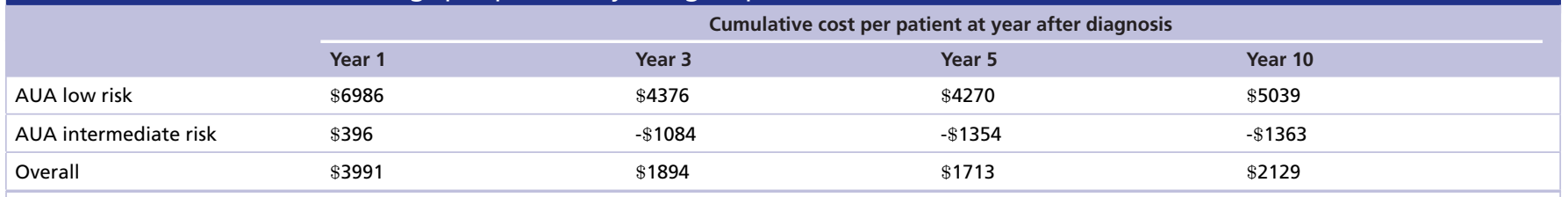

Cost-savings by AUA risk group. The cumulative cost-savings per patient was modeled for AUA low- and intermediate-risk groups. Positive numbers represent cost-savings whereas negative numbers represent cost increases. Although the AUA intermediate-risk group costs increase in year 3 and onward, it is possible that results would differ if the intermediate-risk group was broken out into 'favorable' and 'unfavorable' risk groups, as is commonly done in clinical practice. This distinction was not modeled in the current study because extensive data on the treatment mix for these subgroups are not yet available to support robust model inputs.

AUA: American Urological Association.

\begin{tabular}{|c|c|c|c|c|}
\hline Parameters & $\begin{array}{l}\text { Localized aua low- and } \\
\text { intermediate-risk prostate } \\
\text { cancer patients ( } \mathrm{n})\end{array}$ & $\begin{array}{l}\text { Cumulative cost at year } 3 \text { in } \\
\text { the typical practice scenario }\end{array}$ & $\begin{array}{l}\text { Cumulative cost at year } 3 \text { in } \\
\text { the prolaris scenario }\end{array}$ & $\begin{array}{l}\text { Cumulative savings with prolaris } \\
\text { at year } 3\end{array}$ \\
\hline Per tested patient & 1 & $\$ 20,797$ & $\$ 18,903$ & $\$ 1894$ \\
\hline $\begin{array}{l}\text { 1-million member commercial } \\
\text { health plan }\end{array}$ & 474 & $\$ 9,857,686$ & $\$ 8,959,825$ & $\$ 897,861$ \\
\hline $\begin{array}{l}\text { 5-million member commercial } \\
\text { health plan }\end{array}$ & 2370 & $\$ 49,288,431$ & $\$ 44,799,127$ & $\$ 4,489,303$ \\
\hline \multicolumn{5}{|c|}{$\begin{array}{l}\text { Economic impact of the test. Total number of patients with localized prostate cancer and AUA low- or intermediate-risk disease in the plan. The number of patients with AUA low- and } \\
\text { intermediate-risk disease per health plan is based on published rates of the incidence of prostate cancer in the general population and published stage distribution rates. The Prolaris } \\
\text { Scenario assumes } 100 \% \text { of low- and intermediate-risk health plan members receive the Prolaris test. Total cost of managing all of the patients in the plan with localized prostate cancer in } \\
\text { the typical practice scenario at year } 3 \text {. Total cost of managing all of the patients in the plan with localized prostate in the Prolaris Scenario at year } 3 \text {. Cumulative savings per patient tested } \\
\text { at year } 3 \text {. } \\
\text { AUA: American Urological Association. }\end{array}$} \\
\hline
\end{tabular}

cost-savings that plans would achieve after using the test in a single-incident patient cohort based on a commercial health plan size of 1 million or 5 million members.

\section{Sources of savings}

Figure 1 demonstrates the cumulative cost-savings by source, modeled over 3 years. With the significant increase in the utilization of AS in the modeled cohort, there was an average reduction of $\$ 7966$ in upfront treatment costs per patient. In addition, there was an average cost-savings of $\$ 932$ from definitive treatment follow-up and downstream treatment of advanced disease over the 3-year period. These cost-savings were offset by the addition of AS costs, which averaged $\$ 1416$ per patient, as well as costs associated with the definitive treatment of patients that fail AS, which averaged $\$ 2188$ per patient. Finally, after accounting for the cost of the test $(\$ 3400)$, the resultant cost-savings was approximately $\$ 1894$ per patient tested.

\section{Sensitivity analysis}

No single input, when changed within a reasonable range of values, caused the model to project that the test was no longer cost-saving (Figure 2). The results of the sensitivity analysis are shown in Figure 2. Several key assumptions drove the model, including, the percentage of AUA low-risk patients managed by AS in the typical practice scenario, the cost of RP, the percentage markup that private payers pay relative to Medicare for certain procedures and the cost of RT. The model was also driven by the number of biopsies performed per year for patients managed by AS and the percentage of AUA low-risk patients that adhere to the recommendation for AS after receiving the test.

\section{Discussion}

Previous economic analyses have demonstrated that increasing the use of AS can result in savings compared with single-modality therapy, but this option has not been universally embraced and fully implemented in clinical practice due in part to the difficulty of properly selecting patients with less aggressive disease. In addition, AS is not the optimal approach for all patients or even all low-risk patients. In a subset of patients, prostate cancer can progress quickly if left untreated. Unfortunately, the tools historically used to risk-stratify patients are insufficient in their ability to identify patients likely to experience disease progression. Therefore, physicians are still in need of additional information when determining which treatment path to recommend to their patients. 


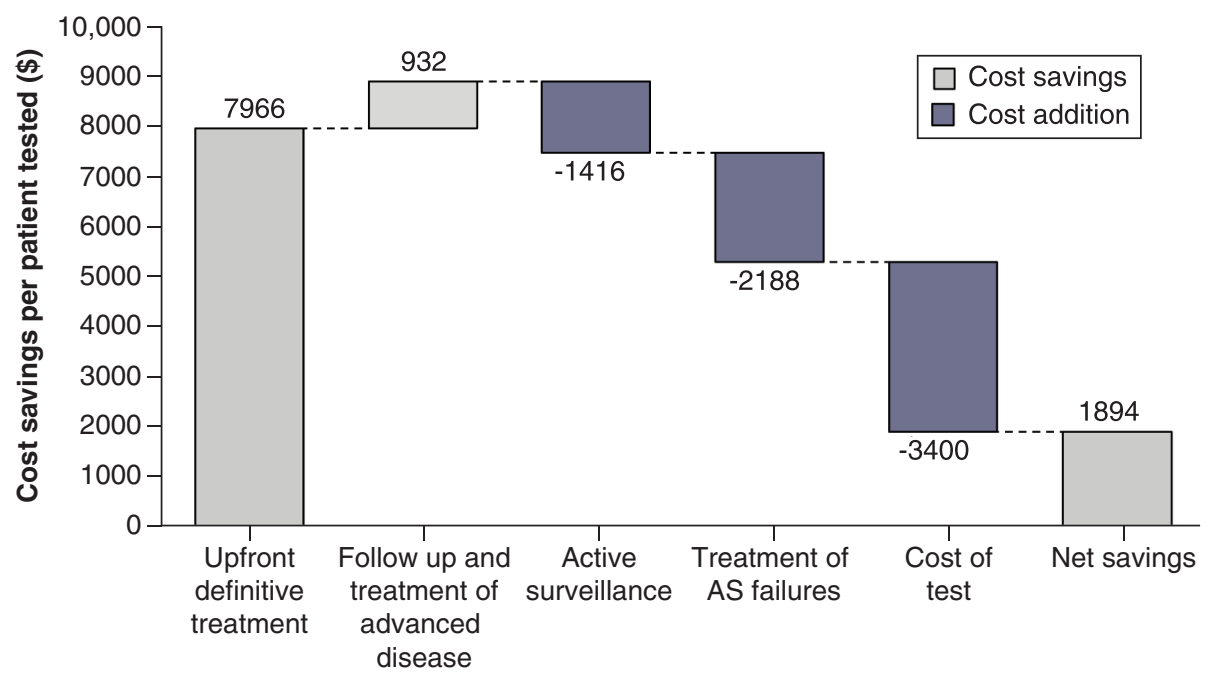

Cost drivers

Figure 1. Source of model savings. Source of model savings. Analysis of the factors in the management of AUA lowand intermediate-risk prostate cancer patients that resulted in cost increases or cost-savings within the model. Positive numbers represent cost reductions whereas negative numbers represent cost increases. AS: Active surveillance; AUA: American Urological Association.

\section{Model input}

AUA low-risk patients managed by AS in typical practice scenario (\%)

Cost of radical prostatectomy

Medicare rate adjustment for private payers

Cost of radiation therapy

Number of biopsies per year for patients managed by AS

AUA low-risk patients adhering to AS recommendation (\%)

AUA low-risk patients managed by AS progressing to Tx (\%)

AUA int-risk patients managed by AS in typical practice scenario (\%)

AUA int-risk patients adhering to AS recommendation (\%)
(A) Base case Conservative input

$23.6 \%$

$\$ 10,604$

$\$ 5800$

$+25 \%$

$+0 \%$

$\$ 34,116$

$-20 \%$

0.5

1.0

$82.4 \%$

$70 \%$

$33 \%$

$40 \%$

$4 \%$

$10 \%$

$82.4 \%$

$65 \%$
Per patient cost savings (\$)

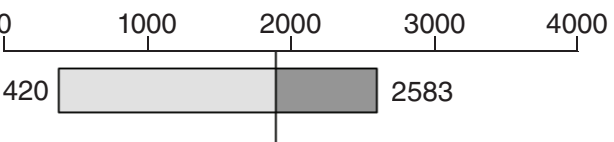

3293

2953

835

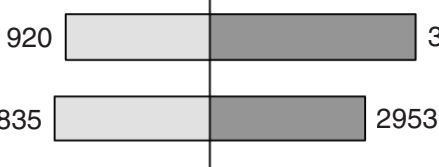

2598

2345

0.25

$90 \%$

$20 \%$

$0 \%$

$90 \%$

(C) Aggressive input

$15 \%$

$\$ 17,500$

$+50 \%$

$+20 \%$

993

2569

2418

2028

2004 
Prolaris is a gene expression assay designed to provide additional information on the aggressiveness of a patient's prostate cancer in order to help physicians and patients determine the best treatment option. Previous studies have shown that utilizing this cell cycle gene expression assay to triage prostate cancer patients often results in a change in clinical treatment decisions and increase in the use of AS [14,15,28,56]. The economic analysis presented here demonstrates that these changes in treatment patterns would also result in significant short- and long-term cost-savings compared with use of standard clinicopathologic tools.

Much of the reduction in cost to payers is derived from the increased use of AS in the subset of AUA low-risk patients who have less aggressive diseases. Given that AUA low-risk patients account for nearly half of patients with incident-localized prostate cancer, this represents a significant opportunity for savings. Additional savings are also accrued through the increased use of AS in intermediate-risk patients. These savings are realized in the first year and reverse over time, due to the higher rate of AS failure in AUA intermediate-risk patients. Despite this, there is still an overall cost-savings per patient when the distribution rate of AUA risk groups in the general population is accounted for.

Incorporation of the cell cycle progression gene expression assay is projected to result in a reduction in the overall cost to payers of treating a cohort of patients with prostate cancer, even after accounting for added costs, including the cost of the test and the cost of biopsies and office visits associated with AS. Per-patient cost-savings are greatest to payers within the first year after patients are tested and placed on AS because the bulk of the cost-savings is derived from the reduction in the upfront costs of definitive treatment. The cumulative cost-savings decline after the first year due to the interplay between cost-savings associated with follow-up for definitive treatment and treatment of advanced disease and the cost additions associated with patients that ultimately fail AS and are placed on definitive treatment.

\section{Limitations}

Although several limitations exist when modeling real-world costs associated with clinical care using an in silico model, this model benefited from the availability of real-world clinical utility data demonstrating how clinicians use the results of the test to guide treatment. Many in silico models have been built based on the assumption that physicians will adhere perfectly to the results of the test when treating patients. However, it is well recognized that compliance in real-world practice is not always the case due to a multitude of other clinical factors at play. For this analysis, populating the model with real-world data on adherence to the test recommendations for AS enhanced the robustness of the output and further supports validates the likelihood that the savings are transferable to a real-world setting. Furthermore, the AS adherence and failure rates used in this model were conservative, as it is reasonable to hypothesize that these rates would further improve in a real-world setting with increased confidence in the cell cycle risk score's stratification and predictive capabilities. For example, this model does not take into account that fewer patients may progress from AS to definitive treatment in the Prolaris Scenario due to patient choice and because they would have lower average predicted risks.

This study is subject to a number of limitations. Recognizing that the quality and reliability of any budgetimpact model is directly related to the data used to generate it, this analysis used both an extensively sourced clinical paradigm and associated cost inputs based on validated reimbursement practices. However, the data are based on a hypothetical cohort of patients and the inputs may therefore not be perfectly representative of a given payer's management of prostate cancer patients. If the clinical paradigm is not reflective of clinical practice within a payer's physician network, the actual cost-savings may differ from the model outputs. Populating the model with data specific to a given patient or provider mix might provide a more accurate representation of the impact of the test for a given payer. For example, RP costs for payers may differ from the conservative inputs used in this study. Indeed, other studies estimating prostatectomy costs based on Medicare reimbursement rates have suggested a range of \$5900-17,500 and these inputs have a significant effect on the cost-savings of the Prolaris Test Scenario, as seen in the sensitivity analysis [57]. Further evaluation of the test using real-world data would provide a more robust understanding of the cost-savings generated in the Prolaris Scenario. Last, clinical data inputs are based on averages and it is therefore difficult to statistically analyze the results of the study.

Another limitation of the model is the decision to exclude nonpayer-related expenses related to prostate cancer. Incorporating these costs into the model would likely serve to increase the cost-savings of the Prolaris Scenario, because a significant increase in AS would result in less of a decrease in productivity in the workplace [58]. In addition, this model does not incorporate the treatment of all potential side effects associated with the treatment 
of prostate cancer, such as the costs associated with the treatment of depression after treatment due to urinary and sexual side effects. If included, these factors would also increase the cost-savings of the Prolaris Scenario.

Other limitations to the model exist. The model assumes utilization of the test in all AUA low- and intermediaterisk patients; use of the test in a different set of patients with prostate cancer could positively or negatively alter the economic impact of the test. Furthermore, clinicians frequently sub-classify intermediate-risk prostate cancer into 'favorable' or 'unfavorable' risk subgroups. This distinction was not modeled in the current study because extensive data on the treatment mix for these subgroups is not yet available to support robust model inputs. As data become available, future studies could build upon the model to assess how the test's clinical utility and associated cost-savings differ between these groups.

Despite these limitations, robust sensitivity analyses around various parameters demonstrated that the model was not driven by a single input or assumption, indicating that even moderate discrepancies between the inputs used to populate the model and those associated with a given payer would still result in substantial cost-savings. This was true even when more conservative inputs were modeled for key parameters. For example, even when higher baseline AS rates were used in the typical practice scenario, the Prolaris Scenario still resulted in cost-savings. One study that analyzed data from The Surveillance, Epidemiology and End Results Program has suggested that AS use is higher than the inputs used in the current model, with approximately $42 \%$ of AUA low- and $10 \%$ of AUA intermediate-risk patients utilizing AS in 2015 [7]. While modeling these conservative inputs for the typical practice scenario did have a significant impact on the cost-savings, neither individually resulted in the Prolaris scenario no longer being cost saving. This finding was also true when both values (for AUA low- and intermediate-risk rates) were input to the model. Furthermore, it is plausible that adherence to a recommendation of AS in the AUA intermediate-risk group should be lower than adherence in the low-risk group, given the increased clinical risk and patient anxiety of the group. Regardless, the sensitivity analyses demonstrated that the cost-savings were not substantially affected by a more conservative input of $65 \%$ adherence that some reports have suggested for the intermediate-risk group [59].

\section{Conclusion}

The health economic evaluation of the impact of the cell cycle gene expression assay demonstrates that use of the test within a US commercial health plan has the potential to result in cost-savings to payers. These savings are primarily the result of increased use of AS in low- and intermediate-risk patients with less aggressive disease. Further studies that assess the impact of the assay in collaboration with third-party payers could be performed to validate these findings and refine the economic impact for a given health plan in a real-world setting.

\section{Summary points}

- Prostate cancer represents a significant portion of cancer spending in the USA today.

- A key driver of these costs is the widespread use of immediate surgical and radiation treatment for the majority of patients upon diagnosis.

- Prior studies have established that broader incorporation of active surveillance, guided by additional prognostic tools, may mitigate the growing economic burden of localized prostate cancer in the USA.

- This study sought to further explore the potential of a particular gene expression-based prognostic tool to address this unmet need.

- A deterministic, decision-analytic model was developed to estimate the economic impact of the Prolaris test on a US commercial health plan.

- When adopted in American Urological Association low- and intermediate-risk patients, the cell cycle gene expression assay was projected to reduce costs by $\$ 1894$ per patient tested over 3 years, growing to $\$ 2129$ per patient over 10 years, after accounting for the cost of the test itself.

- For a large health plan with 5 million members, this would translate to approximately $\$ 4.5$ million in savings over 3 years, which is the average time period commercial insurers are most focused on an intervention's impact given frequency of member churn.

- The majority of savings was derived from reduced use of initial definitive treatment as a result of increased use of active surveillance.

- When employed in clinical practice under the assumptions used in this study, the cell cycle gene expression assay reduces the overall cost of managing prostate cancer patients by better tailoring patient management to disease aggressiveness. 


\section{Author contributions}

G Gustavsen performed conceptualization of study, project administration, supervision, methodology, writing - original draft preparation, writing - review and editing. K Taylor performed data curation, formal analysis, investigation and methodology, writing - review and editing. D Cole performed data curation, formal analysis, investigation, methodology and writing - review and editing. $\mathrm{L}$ Gullet performed data curation, formal analysis, investigation, methodology and writing - review and editing. $\mathrm{N}$ Lewine performed data curation, formal analysis, investigation, methodology and writing - review and editing.

\section{Financial \& competing interests disclosure}

Myriad Genetic Laboratories, Inc. assisted in the funding of this research. The authors have no other relevant affiliations or financial involvement with any organization or entity with a financial interest in or financial conflict with the subject matter or materials discussed in the manuscript apart from those disclosed.

No writing assistance was utilized in the production of this manuscript.

\section{Open access}

This work is licensed under the Attribution-NonCommercial-NoDerivatives 4.0 Unported License. To view a copy of this license, visit http://creativecommons.org/licenses/by-nc-nd/4.0/

\section{References}

Papers of special note have been highlighted as: $\bullet$ of interest

1. Siegel RL, Miller KD, Jemal A. Cancer statistics, 2016. CA Cancer J. Clin. 66(1), 7-30 (2016).

2. Mariotto AB, Yabroff KR, Shao Y, Feuer EJ, Brown ML. Projections of the cost of cancer care in the United States: 2010-2020. J. Natl Cancer Inst. 103(2), 117-128 (2011).

3. American Urological Association. Castration-resistant prostate cancer amended 2018. (2013). www.auanet.org/guidelines/prostate-cancer-castration-resistant-guideline

4. Maurice MJ, Kim SP, Abouassaly R et al. Current status of prostate cancer diagnosis and management in the United States. JAMA Oncol. 2(11), 1505-1507 (2016).

5. Maurice MJ, Abouassaly R, Kim SP, Zhu H. Contemporary nationwide patterns of active surveillance use for prostate cancer. JAMA Intern Med. 175(9), 1569-1571 (2015).

6. Womble PR, Montie JE, Ye $\mathrm{Z}$ et al. Contemporary use of initial active surveillance among men in Michigan with low-risk prostate cancer. Eur. Urol. 67(1), 44-50 (2015).

7. Mahal BA, Butler S, Franco I et al. Use of active surveillance or watchful waiting for low-risk prostate cancer and management trends across risk groups in the United States, 2010-2015. JAMA Letters 321(7), 704-706 (2019).

8. Lawrentschuk N, Klotz L. Active surveillance for low-risk prostate cancer: an update. Nat. Rev. Urol. 8(6), 312-320 (2011).

9. Wilt TJ, Brawer MK, Jones KM et al. Radical prostatectomy versus observation for localized prostate cancer. N. Engl. J. Med. 367(3), 203-213 (2012).

10. Cooperberg MR, Ramakrishna NR, Duff SB et al. Primary treatments for clinically localized prostate cancer: a comprehensive lifetime cost-utility analysis. BJU Int. 111(3), 437-450 (2013).

11. Voigt JD, Dong Y, Linder V, Zappala S. Use of the 4Kscore test to predict the risk of aggressive prostate cancer prior to prostate biopsy: overall cost-savings and improved quality of care to the US Healthcare System. Rev. Urol. 9(1), 1-10 (2017).

12. Keegan KA, Dall'Era MA, Durbin-Johnson B, Evans CP. Active surveillance for prostate cancer compared with immediate treatment: an economic analysis. Cancer 118(14), 3512-3518 (2012).

13. Welch HG, Albertsen PC. Prostate cancer diagnosis and treatment after the introduction of prostate-specific antigen screening: 1986-2005. J. Natl Cancer Inst. 101(19), 1325-1329 (2009).

14. Cuzick J, Swanson GP, Fisher G et al. Prognostic value of an RNA expression signature derived from cell cycle proliferation genes in patients with prostate cancer: a retrospective study. Lancet Oncol. 12(3), 245-255 (2011).

15. Cuzick J, Berney DM, Fisher G et al. Prognostic value of a cell cycle progression signature for prostate cancer death in a conservatively managed needle biopsy cohort. Br. J. Cancer 106(6), 1095-1099 (2012).

16. Cooperberg MR, Simko JP, Cowan JE et al. Validation of a cell-cycle progression gene panel to improve risk stratification in a contemporary prostatectomy cohort. J. Clin. Oncol. 31(11), 1428-1434 (2013).

17. Freedland SJ, Gerber L, Reid J et al. Prognostic utility of cell cycle progression score in men with prostate cancer after primary external beam radiation therapy. Int. J. Radiat. Oncol. Biol. Phys. 86(5), 848-853 (2013).

18. Bishoff JT, Freedland SJ, Gerber L et al. Prognostic utility of the cell cycle progression score generated from biopsy in men treated with prostatectomy. J. Urol. 192(2), 409-414 (2014). 
19. Cuzick J, Stone S, Fisher G et al. Validation of an RNA cell cycle progression score for predicting death from prostate cancer in a conservatively managed needle biopsy cohort. Br. J. Cancer 113(3), 382-389 (2015).

20. Koch MO, Cho JS, Kaimakliotis HZ et al. Use of the cell cycle progression (CCP) score for predicting systemic disease and response to radiation of biochemical recurrence. Cancer Biomark. 17(1), 183-188 (2016).

21. Tosoian J, Chappidi MR, Bishoff JT et al. Prognostic utility of biopsy-derived cell cycle progression score in patients with NCCN low-risk prostate cancer undergoing radical prostatectomy: implications for treatment guidance. BJU Int. 120(6), 808-814 (2017).

22. Lin DW, Crawford ED, Keane T et al. Identification of men with low-risk biopsy-confirmed prostate cancer as candidates for active surveillance. Urol. Oncol. 36(6), 310.e7-310.e13 (2018).

- Validates the active surveillance threshold for the clinical cell-cycle risk score.

23. Leon P, Cancel-Tassin G, Drouin S et al. Comparison of cell cycle progression score with two immunohistochemical markers (PTEN and Ki-67) for predicting outcome in prostate cancer after radical prostatectomy. World J. Urol. 36(9), 1495-1500 (2018).

24. Canter DJ, Reid J, Latsis $\mathrm{M}$ et al. Comparison of the prognostic utility of the cell cycle progression score for predicting clinical outcomes in African American and Non-African American men with localized prostate cancer. Eur. Urol. 75(3), 515-522 (2018).

25. Canter DJ, Freedland S, Rajamani S et al. Analysis of the prognostic utility of the cell cycle progression (CCP) score generated from needle biopsy in men treated with definitive therapy. Prostate Cancer PD 23, 102-107 (2019).

26. Crawford ED, Scholz MC, Kar AJ et al. Cell cycle progression score and treatment decisions in prostate cancer: results from an ongoing registry. Curr. Med. Res. Opin. 30(6), 1025-1031 (2014).

27. Shore ND, Kella N, Moran B et al. Impact of the cell cycle progression test of physician and patient treatment selection for localized prostate cancer. J. Urol. 195(3), 612-618 (2016).

28. Garlich HM. Expanding options in the treatment of prostate cancer: the impact of prognostic biomarkers on patient outcomes. $J$. Manag. Care Med. 23(1), 49-53 (2020).

29. Malone B. Molecular diagnostics reimbursement in flux. Clinical laboratory news. Am. Assoc. Clin. Chem. 39, (2013). www.aacc.org/publications/cln/articles/2013/january/molecular

30. Gustavsen G, Gullet L, Cole D et al. Economic burden of illness associated with localized prostate cancer in the United States. Future Oncol. 16(1), 4265-4276 (2019).

- An up-to-date analysis of the economic burden of localized prostate cancer across different methods of initial management over time.

31. Ip S, Dahabreh I, Chung M et al. An evidence review of active surveillance in men with localized prostate cancer. AHRQ Publication 12, $1-341$ (2011).

32. Klotz L. Active surveillance for low-risk prostate cancer: an update. Nat. Rev. Urol. 8(6), 312-320 (2011).

33. Knighton AJ, Belnap T, Brunisholz K, Huynh K, Bishoff J. Using electronic health record data to identify prostate cancer patients that may qualify for active surveillance. EGEMS 4(3), 1220 (2016).

34. Cooperberg MR. The current management of prostate cancer in the United States: data from the AQUA registry. Presented at: American Urological Association Annual Meeting. MA, USA (12-16 May 2017).

- A comprehensive review of current patterns of care for localized prostate cancer in the community.

35. Maurice MJ, Kim SP, Abouassaly R. Current status of prostate cancer diagnosis and management in the United States. JAMA Oncol. 2(11), 1505-1507 (2016).

- A widespread analysis from the US National Cancer Database establishing a national perspective on the frequency of active surveillance across risk groups.

36. Barocas DA, Cowan JE, Smith JA Jr, Carroll PR. What percentage of patients with newly diagnosed carcinoma of the prostate are candidates for surveillance? An analysis of the CaPSURE database. J. Urol. 180(4), 1330-1334 (2008).

37. Ghia AJ, Shrieve DC, Tward JD. Adjuvant radiotherapy use and patterns of care analysis for margin-positive prostate adenocarcinoma with extracapsular extension: postprostatectomy adjuvant radiotherapy: a SEER analysis. Urology 76(5), 1169-1174 (2010).

38. Cooperberg MR, Lubeck DP, Mehta SS, Carroll PR. Time trends in clinical risk stratification for prostate cancer: implications for outcomes (data from CaPSURE). J. Urol. 170(6 Pt 2), S21-25(2003).

39. Cooperberg MR, Broering JM, Carroll PR. Time trends and local variation in primary treatment of localized prostate cancer. J. Clin. Oncol. 28(7), 1117-1123 (2010).

40. Cooperberg MR, Lubeck DP, Meng MV, Mehta SS, Carroll PR. The changing face of low-risk prostate cancer: trends in clinical presentation and primary management. J. Clin. Onc. 22(11), 2141-2149 (2004).

41. Kaul S, Wonjo KJ, Stone $\mathrm{S}$ et al. Clinical outcomes in men with prostate cancer who selected active surveillance using a clinical cell cycle risk score. Per. Med. 16(6), 491-499 (2019).

- An important study confirming the clinical utility of the clinical cell-cycle risk score.

42. Song J, Li M, Zagaja GP et al. Intraoperative frozen section assessment of pelvic lymph nodes during radical prostatectomy is of limited value. BJU Int. 106(10), 1463-1467 (2010). 
43. Wiegel T, Lohm G, Bottke D et al. Achieving an undetectable PSA after radiotherapy for biochemical progression after radical prostatectomy is an independent predictor of biochemical outcome-results of a retrospective study. Int. J. Radiat. Oncol. Biol. Phys. 73(4), 1009-1016 (2009).

44. Resnick MJ, Koyama T, Fan K-H et al. Long-term functional outcomes after treatment for localized prostate cancer. N. Engl. J. Med. 368(5), 436-445 (2013).

45. Fryar CD, Gu Q, Ogden CL, Flegal KM. Anthropometric reference data for children and adults: United States, 2011-2014. National Center for Health Statistics. Vital Health Stat. 3(39), 9 (2016).

46. MEDPAC. 2011 report to the Congress: medicare and the health care delivery system. http://medpac.gov/docs/default-source/reports /Jun11_EntireReport.pdf

47. Ryan CJ, Smith MR, de Bono JS et al. Abiraterone in metastatic prostate cancer without previous chemotherapy. N. Engl. J. Med. 368(2), 138-148 (2013).

48. Petrylak DP, Tangen CM, Hussain MH et al. Docetaxel and estramustine compared with mitoxantrone and prednisone for advanced refractory prostate cancer. N. Engl. J. Med. 351(15), 1513-1520 (2004).

49. Scher HI, Fizazi K, Saad F et al. Increased survival with enzalutamide in prostate cancer after chemotherapy. N. Engl. J. Med. 367(13), 1187-1197 (2012).

50. De Bono JS, Oudard S, Ozguroglu M et al. Prednisone plus cabazitaxel or mitoxantrone for metastatic castration-resistant prostate cancer progressing after docetaxel treatment: a randomized open-label trial. Lancet 376(9747), 1147-1154 (2010).

51. Fizazi K, Carducci M, Smith M et al. Denosumab versus zoledronic acid for treatment of bone metastases in men with castration-resistant prostate cancer: a randomised, double-blind study. Lancet 377(9768), 813-822 (2011).

52. Paller CJ, Carducci MA, Philips GK. Management of bone metastases in refractory prostate cancer-role of denosumab. Clin. Interv. Aging. 7, 363-372 (2012).

53. SEER Program National Cancer Institute (U.S.). National Center for Health Statistics (U.S.), National Cancer Institute (U.S.), Surveillance Program National Cancer Institute (U.S.), Cancer Statistics Branch, Cancer Control Research Program. SEER Stat Fact Sheets: prostate. NIH Publication 2012. http://seer.cancer.gov/statfacts/html/prost.html

54. Sommers BD, Gourevitch R, Maylone B et al. Insurance Churning Rates For Low-Income Adults Under Health Reform: Lower Than Expected But Still Harmful For Many. Health Affairs 35(10), 1816-1824 (2016).

55. Moody K. Survey: consumers switch health plans often. FierceHealthcare. (2016). www.fiercehealthcare.com/payer/survey-consumers-switch-health-plans-often

56. Cooperberg MR, Simko JP, Cowan JE et al. Validation of a cell-cycle progression gene panel to improve risk stratification in a contemporary prostatectomy cohort. J. Clin. Oncol. 31(11), 1428-1434 (2013).

57. Sharma V, Wymer KM, Borah BJ et al. Cost-effectiveness of active surveillance, radical prostatectomy and external beam radiotherapy for localized prostate cancer: an analysis of the ProtecT trial. J. Urology 22, 964-972 (2019).

58. Venderbos LD, van den Bergh RC, Roobol MJ et al. A longitudinal study on the impact of active surveillance for prostate cancer on anxiety and distress levels. Psychooncology 24(3), 348-354 (2014).

59. Hu JC, Tosoian JJ, Qi J et al. Clinical utility of gene expression classifiers in men with newly diagnosed prostate cancer. JCO Precision Oncol. 2, 1-15 (2018). 

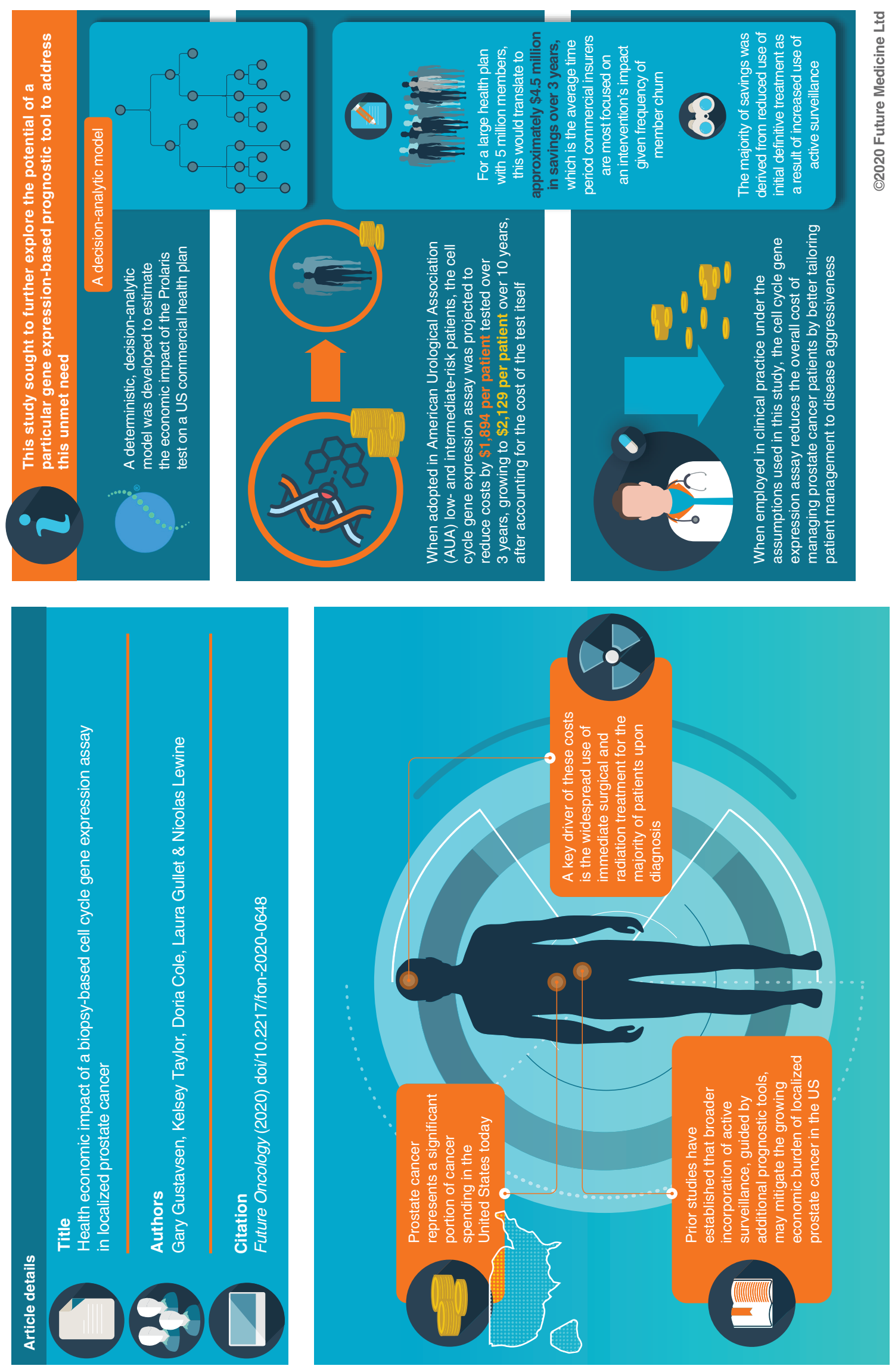